schen Elementen versehen würden. Daher schlägt er vor, im Fall Osteuropas in der Regel nicht von links- oder rechtsextremistischen Parteien, sondern schlicht von extremistischen Parteien zu sprechen.

Unabhängig von diesem Ergebnis und der sich hier aufdrängenden, grundsätzlichen Frage der Einschätzung des Extremismuskonzepts lässt sich die Bewertung Thiemes im Detail nicht immer nachvollziehen. Zum Beispiel spricht er bereits bei der Erfüllung eines Kriteriums für Linksextremismus auf der Ebene der aktuellen Konflikte durch eine Partei von einer „zugleich rechts- und linksextremen Demokratiebedrohung“ und bezeichnet sie als „rechtslinks durchmischte extremistische Vereinigung“ (S. 235). Wenn er etwa angesichts der Tatsache, dass für manche der untersuchten Parteien im Kampf gegen Kriminalität der Zweck die Mittel heiligt und für öffentliche Sicherheit rechtsstaatliche Prinzipien wie das Primat der Unschuldsvermutung sowie der Schutz der Privatsphäre zu vernachlässigen seien und mit Hilfe eines staatlichen Sicherheitsapparates durchgesetzt werden sollen, diese „Forderungen nach Unterordnung des Einzelnen unter den Absolutheitsanspruch des staatlichen Kollektivs“ als „per se linksextreme Ziele“ (S. 235) bezeichnet, so löst dies Verwunderung aus, denn gerade der Ruf nach law-and-order gilt doch als Kernelement extrem rechter Politik.

Wer sich über aktuelle Entwicklungen in osteuropäischen Parteiensystemen informieren möchte oder einen informativen Einblick in ein wenig bearbeitetes Forschungsfeld gewinnen möchte, dem seien die Bücher von Bos und Segert sowie Thieme trotz der genannten Kritikpunkte zu empfehlen.

Jan Schedler und Karsten Schmitz

\title{
Der Wehrbeauftragte des Deutschen Bundestages: nicht angemessen beurteilt
}

Rosenow, Dörthe: Der Wehrbeauftragte im Transformationsprozess. Vom Kontrolleur zum Ombudsmann (Studien der Hessischen Stiftung Friedens- und Konfliktforschung, Band 3), Nomos Verlagsgesellschaft, Baden-Baden 2008, 162 Seiten, € 24,-.

Mit Dörthe Rosenows Buch, entstanden im Rahmen eines von der Hessischen Stiftung Friedens- und Konfliktforschung durchgeführten Forschungsprojekts, über das „Bild vom demokratischen Soldaten“, wird erstmals wieder seit längerer Zeit eine politikwissenschaftliche Abhandlung über das Amt des Wehrbeauftragten (WB) des Deutschen Bundestages vorgelegt. Die Studie gliedert sich in insgesamt sechs Kapitel. Zunächst wird ein knapper Abriss über das Amt des WB, seine Geschichte, Struktur und Aufgaben vorangestellt. Daran schließen sich Ausführungen zu den geänderten Rahmenbedingungen für die Bundeswehr und damit auch für das Amt des WB nach 1990 an, sowie eine theoretische Einbettung der Thematik, in deren Mittelpunkt die Hypothese der „Betriebsblindheit“ des WB steht. Der Hauptteil der Arbeit stützt sich auf die Auswertung der Jahresberichte der WB zwischen 1990 und 2005 sowie auf Interviews mit (ehemaligen) WB und auf Gespräche aus dem Arbeitsumfeld dieser Institution. Zum Abschluss werden die eingangs gestellten Hypothesen überprüft. 
Der mit dem Aufbau von Streitkräften verbundene Machtzuwachs der Exekutive machte in den 1950er Jahren weitergehende parlamentarische Kontrollmöglichkeiten notwendig, um das nach der Verfassung gebotene Gleichgewicht zwischen Parlament und Regierung sicherzustellen. Diesem Bedürfnis nach verstärkten Kontrollmöglichkeiten hat der Gesetzgeber dadurch entsprochen, dass er zwei zusätzliche Institutionen geschaffen hat: den Verteidigungsausschuss, der nach Art. 45a Abs. 2 GG die Rechte eines Untersuchungsausschusses hat, und den nach schwedischem Vorbild gestalteten Wehrbeauftragten (Art. 45b GG). Gerade das Amt des WB, der jeweils für fünf Jahre vom Bundestag gewählt wird, hat die Verabschiedung der verfassungsrechtlichen Grundlagen für den Aufbau der Bundeswehr erleichtert.

Tätig werden kann der WB erstens auf Weisung des Bundestages oder des Verteidigungsausschusses sowie zweitens nach pflichtgemäßem Ermessen, wenn ihm bei der Wahrnehmung seines Truppenbesuchsrechtes, durch Mitteilung von Mitgliedern des Bundestages, Eingaben von Soldaten oder auf andere Weise Umstände bekannt werden, die auf eine Verletzung der Grundrechte der Soldaten oder der Grundsätze der inneren Führung schließen lassen. Wichtigster dieser Grundsätze ist das Leitbild des „Staatsbürgers in Uniform“: Auch der Soldat hat trotz seines besonderen Dienst- und Abhängigkeitsverhältnisses stets Anspruch auf Gewährleistung seiner staatsbürgerlichen und persönlichen Grundrechte und auf Achtung seiner Menschenwürde. Daneben ist das Primat des Politischen und der politischen Führung gegenüber dem Militär und der militärischen Führung hervorzuheben.

Zur Erfüllung seiner Aufgaben kann der WB sich auf eine Reihe von Informationsrechten stützen, die Rosenow im Einzelnen dokumentiert. Insbesondere sein Recht, ohne vorherige Anmeldung jederzeit Truppenteile oder Dienststellen zu besuchen, ist die bedeutendste Erkenntnisquelle des WB. In den Worten des amtierenden WB Reinhold Robbe „nimmt nach dem Gesetz der WB seine Aufgaben als Hilfsorgan des Bundestages bei der Ausübung der parlamentarischen Kontrolle wahr. [Darüber hinaus] ist er aber [auch] ,Sachwalter' der Interessen der Angehörigen der Streitkräfte." Das heißt, der WB hat nicht nur eine Kontroll- und Schutzfunktion, sondern auch eine Fürsorgepflicht für die Mitglieder der Streitkräfte, was von allen seit 1990 amtierenden WB in ihren Jahresberichten herausgehoben wird. An dieser Funktion setzt die besondere Kritik Rosenows nach Auswertung der Jahresberichte sowie der Interviews an: In Anlehnung an Badura und Reese ${ }^{1}$ wirft sie den WB „zunehmende Betriebsblindheit“ (S. 55) vor. Da nämlich alle WB seit 1990 „eine langjährige parlamentarische Sozialisation hinter sich haben, kann die These aufgestellt werden, dass eine umfassende Problemsicht und der Kontrollwille gegenüber der Exekutive und damit auch der Bundeswehr bereits während der parlamentarischen Sozialisation verloren gegangen ist" (S. 56). Belegen kann die Autorin diese Vermutung jedoch nicht. Vielmehr versteift sie sich auf die Feststellung, dass es sich „um eine ,Betriebsblindheit' im tieferen Sinne [handelt], weil die umfassende Problemsicht nicht nur aufgrund der Beschäftigung mit Detailproblemen, sondern auch aufgrund der Identifikation mit den individuellen Problemen der einzelnen Soldatin/des einzelnen Soldaten verloren geht“ (S. 98). Diese Einschätzung, dass die WB durch den seit 1990 erfolgten Transformationsprozess ihren Funktionen nicht hinreichend nachkommen, begründet sie mit Einzelfällen, etwa den Ereignissen in der Kaserne in Coesfeld (Demütigung von Untergebenen) und die in ihren

1 Bernhard Badura / Jürgen Reese, Jungparlamentarier in Bonn. Ihre Sozialisation im Deutschen Bundestag, Stuttgart / Bad Cannstatt 1976. 
Augen nicht genug vom damaligen WB gewürdigte Eingabe des Majors Florian Pfaff. Dieser hatte seinen Dienst in der Bundeswehr verweigert, weil er glaubte, die Bundesrepublik würde trotz gegenteiliger Versicherungen durch bestimmte Aktionen, zum Beispiel den Schutz der US-Liegenschaften, die USA im Irak-Krieg unterstützen.

So verdienstvoll die Durchsicht der Jahresberichte seit 1990 sowie die mit den WB geführten Interviews sind, so sehr drängt sich dem Leser der Eindruck auf, dass dies mit dem Ziel geschah, angebliche Defizite zu finden, um die These von der „Betriebsblindheit“ im Amt des WB zu belegen. So ist nicht einsichtig, warum sich die WB seit den Auslandseinsätzen der Bundeswehr nicht verstärkt mit „Detailproblemen“ der Mitglieder der Streitkräfte zu beschäftigen haben, und warum durch diese Fürsorgetätigkeit den WB „die umfassende Problemsicht auf die Institution Bundeswehr verloren“" gehen sollte (S. 142). Bei einer unvoreingenommenen Durchsicht der Jahresberichte kann - wie von Rosenow unterstellt - von einer „Vernachlässigung der Kontrolle der Institution Bundeswehr gegenüber der Zivilgesellschaft“ (S. 142) nicht gesprochen werden. Folglich ist die Interpretation, die Tätigkeit der WB als „betriebsblind“ zu bezeichnen, fragwürdig. Immerhin konzediert die Autorin an einer Stelle dem ehemaligen WB Willfried Penner, dass er „darum bemüht gewesen sei, sein Amt im Interesse des Parlamentes und die Aufgabe der Kontrolle fokussierend auszuüben" (S. 97).

Insgesamt bleibt dennoch der Eindruck einer dem Amt des Wehrbeauftragten und seiner Praxis nicht gerecht werdenden Publikation.

Udo Kempf

\title{
Föderalismusreform I: Ertrag jahrzehntelanger Forschung
}

\author{
Scharpf, Fritz W.: Föderalismusreform. Kein Ausweg aus der Politikverflechtungsfalle? (Schriften \\ aus dem Max-Planck-Institut für Gesellschaftsforschung, Band 64), Campus Verlag, Frankfurt \\ am Main 2009, 174 Seiten, € 24,90.
}

Anzuzeigen ist eine Publikation, die alle aufmerksamen Beobachter des real-existierenden Föderalismus in Deutschland besonders interessieren dürfte: das neueste Buch des Föderalismusexperten Fritz Scharpf. Scharpf, emerierter Direktor des Max-Planck-Instituts für Gesellschaftsforschung, ist nicht nur ein Experte des Regierens im Mehrebenensystem, sondern gilt bekanntlich auch als Urheber der These von der Politikverflechtungsfalle. Das äußerst lesenswerte Buch handelt vornehmlich von der Föderalismusreform, erster Teil, die durch ein verfassungsänderndes Gesetz zum 1. September 2006 ins Werk gesetzt wurde.

Nach einer knappen Einleitung wird das Thema zunächst in historischer Perspektive entwickelt („Die Genealogie der Politikverflechtung“). Darin erfährt der Leser, wie sich das Verhältnis von Bund und Ländern seit 1949 entwickelte und wer für die Zunahme der Zustimmungsgesetze - und damit des Maßes an Politikverflechtung - die Hauptverantwortung trägt: die erste Große Koalition von 1966 bis 1969 und das Bundesverfassungsgericht durch eine großzügige Interpretation der alten Bedürfnisklausel des Art. 72 Abs 2 GG.

Im zweiten Kapitel („Probleme der Verflechtung“) liefert Scharpf den Ertrag seiner jahrzehntelangen Forschungen über das Regieren zwischen Bund und Ländern. Dabei lernt der 